\title{
Albedos of Small Hilda Group Asteroids as Revealed by Spitzer
}

\author{
ERIN LEE RYAN, CHARLES E. WOODWARD \\ Department of Astronomy, School of Physics and Astronomy, 116 Church Street, S. E., \\ University of Minnesota, Minneapolis, MN 55455, \\ ryan@astro.umn.edu,chelsea@astro.umn.edu
}

Received __; accepted _

AJ ver October 29, 2018 


\begin{abstract}
We present thermal $24 \mu \mathrm{m}$ observations from the Spitzer Space Telescope of 62 Hilda asteroid group members with diameters ranging from 3 to 12 kilometers. Measurements of the thermal emission when combined with reported absolute magnitudes allow us to constrain the albedo and diameter of each object. From our Spitzer sample, we find the mean geometric albedo, $p_{V}=0.07 \pm 0.05$ for small $(\mathrm{D}<10 \mathrm{~km})$ Hilda group asteroids. This value of $p_{V}$ is greater than and spans a larger range in albedo space than the mean albedo of large $(\mathrm{D} \gtrsim 10 \mathrm{~km})$ Hilda group asteroids which is $p_{V}=0.04 \pm 0.01$. Though this difference may be attributed to space weathering, the small Hilda group population reportedly displays greater taxonomic range from $\mathrm{C}$-, D- and X-type whose albedo distributions are commensurate with the range of determined albedos. We discuss the derived Hilda size-frequency distribution, color-color space, and geometric albedo for our survey sample in the context of the expected migration induced "seeding" of the Hilda asteroid group with outer solar system proto-planetesimals as outlined in the "Nice" formalism.
\end{abstract}

Subject headings: solar system: minor planets, asteroids: surveys 


\section{INTRODUCTION}

Residing in the outer main belt at a distance of $\simeq 4.04 \mathrm{AU}$ in the first-order Jupiter J3:2 mean resonance is an asteroid population with an unknown origin, the Hilda asteroid group (Gradie et al. 1989). Early dynamical models suggested that the Hilda group originated field asteroids which were captured in gravitational resonances as Jupiter migrated in a sunward direction (e.g., Franklin et al. 2004). In contrast, more expansive models designed to explain the origins of a wide variety of outer solar system small bodies families, the 'Nice Model' (Levison et al. 2009) argues that delivery of proto-Kuiper Belt planetesimals into stable inner solar system orbits, such as exhibited by the Hilda asteroid group, occurred populating the dynamical families observed at the present epoch. Though the Nice Model favors migration from the Kuiper Belt, observational evidence of such small body migration is tentative at best. Could the Hilda group asteroids be the remnant population arising from the purported migration effects detailed in the Nice Model or are the antecedents field asteroids? Answers to this question have a direct bearing on the efficiency of early solar system dynamical processes outlined in the Nice model.

A detailed analysis of the colors and albedos of the Hilda population yields clues to their origin and can substantiate outcomes described by the Nice formalism. Large (diameters $\gtrsim 10 \mathrm{~km}$ ) Hilda asteriods have a range of $V-R$ color magnitude, 0.38 to 0.49 (Dahlgren et al. 1998), which is similar to those colors of comets (Hainaut \& Delsanti 2002). In particular ecliptic comets (ECs) span the same narrow range of $V-R$ (Lamy \& Toth 2009) as the Hildas, and are thought to have originated as outer solar system Centaurs which were subsequently scattered into the inner solar system due to gravitational interactions with Jupiter. C-type asteroids in the outer asteroid belt also inhabit this range of color space. However, the $V-R$ colors of smaller Hildas show greater spectral diversity (Gil-Hutton \& Brunini 2008) commensurate with the colors of both C- and X-asteroid 
taxonomic type as well as some Kuiper Belt objects (Gulbis et al. 2006).

The geometric albedos of large Hilda group asteroids from Rvan \& Woodward (2010) are commensurate with the albedo range for ECs (Lamy et al. 2004) and other icy bodies such as main belt comets (Hsieh et al. 2009). The albedo segregation of cold classical Kuiper Belt objects (KBOs) and hot classical KBOs is argued to indicate differing origins of these two populations (Brucker et al. 2009). If a Nice model-like migration occurred and the small Hilda group asteroids did indeed originate in the proto-Kuiper Belt, we expect that the albedos of these objects would be commensurate with the albedos of the low inclination, cold classical KBOs.

Here, we present an analysis of the albedo and diameter of Hilda family asteroids extracted from the NASA Spitzer (Werner et al. 2004) infrared (IR) archival database, cross referenced with extant optical photometry. In $\$ 2$, generation of the asteroid survey sample is presented, 33 outlines our thermal model analysis of the photometry, \$4 discusses the outcomes of our analysis, including an estimate of the size-frequency distribution inferred from the Hilda asteroid group sample and whether the Hilda group are indeed a migrant population, while concluding remarks are summarized in $\$ 5$,

\section{ARCHIVAL ANALYSIS}

The photometry discussed herein was obtained from the NASA Spitzer Archive and represents all Hilda asteroids observed in Program Identification number (PID) 40819. These data were obtained in Cycle- 4 of the Spitzer cryogenic mission with the Multiband Imaging Photometer instrument (MIPS; Rieke et al. 2004). The MIPS $24 \mu \mathrm{m}$ band imager is a $128 \times 128$ pixel Si:As impurity band conduction detector with an effective wavelength of $23.68 \mu \mathrm{m}$ with a native pixel scale of $2.49 \operatorname{arcsec} \times 2.6 \operatorname{arcsec}$. All $24 \mu \mathrm{m}$ images are 
diffraction limited. All targets in PID 40819 were observed in MIPS Photometry mode; data was only obtained in the 24 and $70 \mu \mathrm{m}$ channels. No useful $70 \mu \mathrm{m}$ data exists due to the offset of $\sim 12$ arcminutes between the two fields of view.

Observations utilizing the Compact Source Photometry (CSP) template in this PID consist of 14 images each with an exposure of $3 \mathrm{sec}$ in length resulting in a total observation time per object of $42 \mathrm{sec}$. Each observation utilized 10.55 mins of spacecraft time including overhead. Basic data processing including removal of dark current, flat fielding, and flux calibration was performed via the automated Spitzer pipeline ver. 18.2.0 (Gordon et al.) 2005) to create basic calibrated data (BCDs).

We used the MOPEX (Makovoz \& Marleau 2005) program to obtain photometry on all 14 BCDs in an image data stack utilizing point-spread function (PSF) photometry while subtracting the median background, and then averaged fluxes and calculated errors. Photometry reported in Table 1 is the average of the 14 BCDs in the stack. Uncertainties reported in Table 1 include the photometric fitting errors reported from MOPEX and the absolute calibration uncertainty of the $24 \mu \mathrm{m}$ channel of order $2 \%$ (Engelbracht et al. 2007) added in quadrature.

\section{THERMAL MODELS}

To determine the diameters and albedos of the Hilda group asteroid in our sample, we have utilized both the Standard Thermal Model (STM; Lebofsky \& Spencer 1989) and the Near Earth Asteroid Thermal Model (NEATM; Harris 1998). The STM and the NEATM both rely upon a basic radiometric method to determine the diameter and albedo of an asteroid (for details see Rvan \& Woodward 2010). Both models assume balance between incident radiation and emitted radiation, where the emitted radiation has two components; 
a reflected and a thermal component. The reflected component has approximately same spectral energy distribution (SED) as the incident radiation; i.e., the reflected component is dominant in the optical and peaks in $\mathrm{V}$ band commensurate with the spectral region in which the sun emits the greatest flux. The reflected asteroid flux is proportional to the diameter of the body, $D(\mathrm{~km})$ and the geometric albedo, $p_{V}$. To maintain energy balance the thermal flux is proportional to the amount of incident flux which is not reflected; $F_{\text {thermal }} \propto D^{2}\left(1-p_{v}\right)$.

However, asteroids do not maintain one single body temperature, $\mathrm{T}(\mathrm{K})$, rather there is a temperature distribution across the surface which is then radiometrically observed in the mid-IR. The STM and the NEATM utilize two different assumed temperature distributions to model the total IR flux, which then yields the geometric albedo. The temperature distributions invoked by each model are expressed as:

$$
T_{S T M}(\Omega)=\left[\frac{(1-A) S_{\odot}}{0.756 r_{h}^{2} \epsilon \sigma}\right]^{\frac{1}{4}}(\cos \Omega)^{\frac{1}{4}}
$$

and

$$
T_{N E A T M}(\phi, \theta)=\left[\frac{(1-A) S_{\odot}}{\eta r_{h}^{2} \epsilon \sigma}\right]^{\frac{1}{4}}(\cos \phi)^{\frac{1}{4}}(\cos \theta)^{\frac{1}{4}}
$$

where the temperature, $\mathrm{T}$ is in Kelvin, $\mathrm{A}$ is the geometric Bond albedo, $\mathrm{S}_{\odot}$ is the solar constant $\left(\mathrm{W} \mathrm{m}^{-2}\right), \mathrm{r}_{h}$ is the heliocentric distance $(\mathrm{AU}), \epsilon$ is the emissivity of the object which is assumed to be 0.9, an appropriate value for rock (Morrison 1973), $\sigma$ is the Stefan-Boltzmann constant, $\Omega$ is the angular distance from the sub-solar point on the asteroid, $\eta$ is the beaming parameter, $\phi$ is the latitude, and $\theta$ is longitude of the coordinate grid superposed on the asteroid.

In the NEATM temperature distribution, $\eta$, the beaming parameter is utilized as 
a varying parameter to characterize both shape and thermal inertia. In an ideal case where an asteroid is a perfect sphere with zero inertia, $\eta=1.0$. Only one thermal $(24 \mu \mathrm{m})$ photometric measurement is available from the Hilda asteroid group measurements (Table 1); therefore, NEATM was run with a fixed beaming parameter of $\eta=0.91$. This value of $\eta$ was selected by averaging the value of $\eta$ for 23 large Hilda group asteroids observed by IRAS and/or MSX derived by Ryan \& Woodward (2010). In addition we adopted a phase slope parameter $(G)$ of 0.15 for the purposes of modeling the PID 40819 asteroid $24 \mu \mathrm{m}$ photometry. To compute the geometric albedo and thus the temperature distribution on the illuminated face of the asteroid, one must anchor solutions with optical radiometric measurements. We utilized absolute magnitudes $(H)$ from the Minor Planet Center 1 (MPC) for this purpose.

All albedo and diameter solutions reported in Table 2 are derived from Monte Carlo modeling. A 500 data point distribution was created for each object observation such that the mean flux was equal to the $24 \mu \mathrm{m}$ flux measured by MOPEX and the standard deviation of the distribution was equivalent to the uncertainties in the flux measurement. These flux points were then used in conjunction the known orbital parameters and the absolute magnitude $(\mathrm{H})$ to produce albedo and diameter fitting results. Due to the wide width of the $24 \mu \mathrm{m}$ channel, a color correction is also required to accurately fit the albedo and diameter. Our implementation of STM and NEATM applies the color corrections iteratively, such that a color correction is applied with each refinement of the albedo (Ryan \& Woodward 2010). Instead of using the subsolar temperature for the color correction, we calculate the mean of the temperature distribution for the application of the color correction, as described in Ryan \& Woodward (2010). With the STM, albedos were computed over the range of color corrections varying the temperatures from 70 to $300 \mathrm{~K}$, to examine the

\footnotetext{
${ }^{1}$ www.cfa.harvard.edu/iau/mpc.html
} 
fidelity of our approach as our color correction methodology differs from other described in

the literature (e.g., Brucker et al. 2009; Stansberry et al. 2007). The albedo deviations over the latter temperature range of color corrections were $\lesssim 2 \%$ indicating that uncertainties in the derived albedo from color corrections are low compared to the uncertainties in the photometry. The albedos and diameters listed in Table 2, are the mean of the 500 Monte Carlo solutions for each asteroid. The standard deviation of these solutions is taken to be the statistical uncertainty $( \pm)$ in the results listed in Table 2 ,

\section{RESULT \& DISCUSSION}

The distinct differences between the albedo distribution of Hilda asteroid group in the IRAS database (Ryan \& Woodward 2010) as opposed to those observed in the MIPS $24 \mu \mathrm{m}$ survey is clearly illustrated in Fig. 1. The mean NEATM geometric albedo of Hildas derived from IRAS photometry is $p_{V}=0.04 \pm 0.01$ whereas, the mean NEATM albedo for asteroids from Spitzer MIPS photometry is $p_{V}=0.07 \pm 0.05$. These two albedo distributions appear similar; however, the distribution for the small asteroids ( $D \lesssim 12 \mathrm{~km}$ ) exhibits a high albedo tail which is not seen in the large $(\mathrm{D}>12 \mathrm{~km})$ asteroid population.

Figures $\left[2\right.$ and 3 illustrate the trend of geometric albedo $\left(p_{V}\right)$ as a function of diameter (in $\mathrm{km}$ ). Notable is the trend that higher albedos are associated with decreasing asteroid diameters. To test the significance of this trend, we have calculated the Spearman rank-order coefficient (e.g., Meyers \& Well 2003) for all Hildas with known albedos from derived from the IRAS archive (Ryan \& Woodward 2010) and this work, as well as the rank-order coefficient for solely the Hildas from the Spitzer MIPS survey (Table 2). The Spearman rank-order coefficient (Spearman $r_{s}$ ) is commonly defined as 


$$
r_{s}=1-\left[\frac{6 \Sigma d_{i}^{2}}{n\left(n^{2}-1\right)}\right]
$$

which assess the statistical dependence between two variables assuming that the relationship can be described by a monotonic function. The $r_{s}$ for the complete, aggregate Hilda asteroid group data set (total population sample $n=85$ ) is -0.69 which corresponds to a probability of non-correlation between diameter and albedo of $3.94 \times 10^{-13}$. The correlation is significant to the $6.3 \sigma$ level. Using only the smaller Hilda population from the Spitzer MIPS data $(n=62), r s=-0.76$ and the probability of non-correlation is $4.54 \times 10^{-13}$ with a correlation significance of $5.97 \sigma$. Whether or not the correlation between albedo and diameter may be described by a monotonic trend may be influenced by the optical survey completeness. However, we assert that the optical detection bias is not the cause of the derived albedo increase at small diameters as elucidated in $\S 4.1$.

Figures 4 and 5 plot albedo as a function of various orbital parameters. No trends are readily apparent between albedo and any of these parameters.

\section{1. $\quad$ Effects of Completeness}

There are two possible sources of completeness that affect the interpretation of the albedo vs. diameter relationship derived from the Hilda asteroid sample, IR completeness and optical completeness. The asteroids in the Hilda sample (Table 1) with the three highest albedos, 2003 SB45, 2005 EC205, 2003 WD111, are also the faintest objects in the Spitzer $24 \mu \mathrm{m}$ sample. From the MIPS Instrument Handbook2, the $1 \sigma$ sensitivity limit for a $42 \mathrm{sec}$. astronomical observing request (AOR) is $214 \mu \mathrm{Jy}$ in a region with high background levels such as those found along the line of sight through the zodical light. The sensitivity

\footnotetext{
${ }^{2}$ http://ssc.spitzer.caltech.edu/mips/mipsinstrumenthandbook/
} 
limit of the MIPS instrument is proportional to the square root of the exposure time in the background limited case. Following this latter convention, the $5 \sigma$ detection limit for a 3 sec. BCD frame is $4004 \mu \mathrm{Jy}$. Nine asteroids are not detected to a $5 \sigma$ level in individual BCDs. Even if these objects are excluded from the Hilda population sample, there is still a trend towards higher albedo with smaller diameter with a correlation significance of $4.8 \sigma$ as determined from the Spearman rank-order test.

The optical completeness for the Hilda asteroid group and the photometric uncertainty associated with the optical data may also bias the derived results. To assess the completeness of asteroid surveys, we assume that they are complete to a $\mathrm{V}$ magnitude of 21.5, commensurate with the completeness limits of the Sloan Digital Sky Survey (Ivezić et al. 2001), the Sub-Kilometer Asteroid Diameter Survey (Gladman et al. 2009), and Spacewatch (Larsen et al. 2007). Assuming that an asteroid will be detected at opposition by one of a number of surveys, the relation $m_{V}=H+5 \log \left[r_{h}\left(r_{h}-1\right)\right]$, can be used to estimate completeness, where we substituting the mean aphelion distance of 4.79 AU derived from the orbital parameters of 3509 known Hilda group asteriods for the heliocentric distance, $r_{h}$. We find that optical surveys must be complete to at least H=15.21 magnitude. Only two objects in Table 1, 2004 TP256 and 2001 UY149, are at this completeness limit or a fainter limit; therefore, our Hilda sample is not dominated by the effects of optical completeness. The albedo and albedo uncertainties in Table 2 are derived solely from the uncertainties in the mid-IR photometry and do not include uncertainties in the $\mathrm{H}$ magnitudes. $\mathrm{A} \pm 0.1$ magnitude uncertainty in $\mathrm{H}$ results in $\mathrm{a} \simeq 10 \%$ variation in the value calculated albedo. Thus, the marked change in slope of albedo-diameter relation evident in Figs. 2 and 3 is likely significant and not an artifact related to sample size or optical completeness effects as the variation is a $\gtrsim 10 \%$ deviation from the derived mean geometric albedo. 


\subsection{Selection of NEATM Beaming Parameter}

The selection of a fixed value for $\eta$ in NEATM may introduce a systematic uncertainty in the derived values for the albedo and diameter. In particular, a linear relation between

$\eta$ and the phase angle has been noted by Delbo et al. (2007) and Wolters et al. (2008) within the Near Earth Asteroid (NEA) population. As the IRAS and Spitzer Hilda asteroid samples used in our analysis contain a range of phase angles between $11^{\circ}$ and $18^{\circ}$, no adjustment to the mean beaming parameter, $\eta=0.91$ (see $\S$ 3) was used nor needed.

The selection of a mean beaming parameter may introduce some error to the fitted albedo and diameter values for these objects. The mean beaming parameter of the large Hilda population is $\eta=0.91$; however, the standard deviation of the beaming parameter for this asteroid population is $=0.14$. Though this variation is smaller than the standard deviation for the main belt as a whole (where the standard deviation of $\eta \sim 0.27$ ), this variation can introduce errors in the albedo and diameter calculations presented in $\S$. To characterize the range of uncertainty we re-analyzed our photometry from Table 1 with NEATM utilizing fixed $\eta$ values of 0.77 and 1.05 . For a value of $\eta=0.77$, the calculated albedos of asteroids are on average $4 \%$ higher and the calculated diameters are $1 \%$ smaller than the values calculated when $\eta=0.91$. If a value of $\eta=1.05$ is used, the calculated albedos of asteroids are on aver $20 \%$ lower and diameters increase by $12 \%$. This increase in beaming parameter subsequently reduces a mean small Hilda asteroid population albedo to $p_{V}=0.053$ which is closer to the mean value for the large Hilda asteroids observed by IRAS. An increase in the beaming parameter would correspond to an increased thermal inertia, which to first order is an inverse function asteroid diameter resulting trend for the Hildas that is self-consistent with that derived for the main belt and Near Earth Asteroid

by Delbo \& Tanga (2009). However, an increase in thermal inertia would require that small Hilda asteroids retain less regolith than large Hildas, which has not yet been observed via 
mid-infrared spectroscopic surveys. Studies of similar primitive type objects such as small Trojan asteroids (Fernandez et al. 2009) and comet nuclei at 4-5 AU (Fernandez et al. 2008) have derived beaming parameters near the value of $\eta=0.91$ indicating that the value used in our study of Hildas is appropriate for primitive outer solar system objects.

\subsection{The Size-Frequency Distribution}

The size-frequency distribution of the Hilda asteroids can be inspected to determine if the group is in collision equilibrium. The modeling of Dohnanyi (1969) indicates that an asteroid population is in collisional equilibrium if the cumulative size-frequency distribution is near a diameter slope of -2.5 .

We derived a best-fit relationship between albedo and diameter using a second-order polynomial of the form

$$
p_{V}=0.04-0.49\left(\frac{1 k m}{D}\right)+4.14\left(\frac{1 k m}{D}\right)^{2}
$$

to determine if the Hildas are in collisional equilibrium, as the relationship appears to be an inverse function of diameter (Figs. 2, 3). For all asteroid diameters $\lesssim 4 \mathrm{~km}$, which are beyond the completeness limit of our sample, $p_{V}$ is held fixed at a value of 0.2 . The albedo function described by Eqn. 4 was used in conjunction with $\mathrm{H}$ magnitudes of all known Hildas to derive a size-frequency distribution, Fig. 6. To illustrate the effect of using an alternative albedo function, we also plot a cumulative size-frequency distribution in Fig. 6 assuming all Hilda group asteroids regardless of diameter have a mean geometric albedo of 0.04 .

Functionally the two size-frequency distributions appear similar at large diameters, and each both appear to break at diameters, $D \lesssim 10 \mathrm{~km}$. The cumulative size-frequency 
distribution calculated with our albedo function can be described by a broken power law of the form $N_{i}=\beta \times D(\mathrm{~km})^{\alpha}$ with two components. For large Hilda group asteroids with $D>12 \mathrm{~km}, \beta=94317 \pm 3395$ and $\alpha=-(2.00 \pm 0.02)$ while for the smaller population, $5<D(\mathrm{~km})<12, \beta=2696 \pm 189, \alpha=-(0.37 \pm 0.08)$. The value of $\alpha=-2.00$ for the larger Hilda asteroid group population strongly suggests that they are in near-collisional equilibrium (Dohnanvi 1969). However, there are less smaller Hilda asteroids observed than expected for such a system. If the cumulative number distribution for diameters $>12 \mathrm{~km}$ was continuous, one would predict a total number of 3775 Hildas with diameters $>5 \mathrm{~km}$, rather than the 1334 Hildas observed with diameters $>5 \mathrm{~km}$.

An explanation for the decrement of small Hilda group asteroids in the size-frequency distribution illustrated in Fig. 6 is a lack of optical completeness. However, the optical data is complete to $\mathrm{H} \simeq 15$ magnitude, corresponding to a diameter of at least $6.6 \mathrm{~km}$ (assuming an albedo of 0.04 ). The inversion in the size-frequency distribution begins near $D \simeq 10 \mathrm{~km}$, far before this small diameter break point. An alternative interpretation is that the shallow slope at small diameters traces the depletion of small objects from the Hildas. Models by Gil-Hutton \& Brunini (2000) suggest that collisions within the Hilda asteroid group population from scattered objects originating in the Uranus-Neptune zone could produce a large number of asteroid fragments with relative velocities high enough to escape the resonance. Thus, the decrement of small Hilda group asteroids could be explained via an intense collisional period, such as occurred in the late Heavy Bombardment epoch in the early solar system followed by a long period of low collisional activity. The age estimate of $\gtrsim 4$ Gyr for the Hilda family (Broz \& Vokrouhlicky 2008), a dynamical subgroup of the Hilda asteroid group (see $\S$ 4.4) lends credence to the later hypothesis.

A lower limit to the total Hilda asteroid group mass can be derived by integrating over the differential size distribution, $n(r) d r$, assuming an average bulk density, $\rho_{\text {Hilda }}^{\text {ave }}$. The 
differential size distribution has two components,

$$
n_{1}(r) d r=47186 \times\left(\frac{1 \mathrm{~km}}{r}\right)^{3.00 \pm 0.02} d r \quad(6 \mathrm{~km} \leq r \leq 90 \mathrm{~km})
$$

and

$$
n_{2}(r) d r=772 \times\left(\frac{1 \mathrm{~km}}{r}\right)^{1.37 \pm 0.08} d r(2.5 \mathrm{~km} \leq r \leq 6 \mathrm{~km})
$$

where $r$ is the asteroid radius. The lower limit of Hilda asteroid group mass is therefore,

$$
M_{T}=\int_{0}^{r_{c}} \frac{4}{3} \pi \rho_{\text {Hilda }}^{\text {ave }} r^{3} n_{2}(r) d r+\int_{r_{c}}^{90} \frac{4}{3} \pi \rho_{\text {Hilda }}^{\text {ave }} r^{3} n_{1}(r) d r
$$

where $n_{1}$ and $n_{2}$ are from Eqns 5 and 6 , $\rho_{\text {Hilda }}^{\text {ave }}=2300 \mathrm{~kg} \mathrm{~m}^{-3}$ is the bulk density, and $r_{c}$ is the radius, $12.5 \mathrm{~km}$, at which the two differential size distributions are equal. We find that $M_{T} \approx 4 \times 10^{19} \mathrm{~kg} \approx 6 \times 10^{-6} \mathrm{M}_{\text {Earth }}$, equivalent to a $165 \mathrm{~km}$ radius sphere having the same density which would be approximately twice as large as 153 Hilda.

\subsection{Origins}

The observed albedo variations that seem diameter dependent likely is indicative of the Hilda asteroid group origins and evolutionary processes. Variation in the relative degree of metamorphism within the parent body population arising from differences in the extent of internal melting, caused by the radioactive decay nucleotides such as ${ }^{26} \mathrm{Al}$ or ${ }^{60} \mathrm{Fe}$, might yield a population dichotomy in the currently observed Hilda asteroid group (McCoy, Mittlefehldt, \& Wilson 2006). Asteroids with high albedos arise perhaps from the most thermally altered bodies in this primordial population. However, the Hilda asteroid group sampled in our MIPS survey likely is in collisional equilibrium for asteroids with 
diameters $>10 \mathrm{~km}$ and thus we do not expect to see such a clear trend of albedo with diameter as evident in our results. We also do not expect a thermally modified parent body in the region of the Hilda group as the models of Grimm \& McSween (1993) indicate that a sufficent quantity of ${ }^{26} \mathrm{Al}$ necessary to cause internal melt would not be accreted in planetesimal bodies of all sizes formed at heliocentric distances $\gtrsim 3.4$ AU.

The discovery of distinct dynamical families within the Hilda group by Broz \& Vokrouhlicky (2008) necessitates examination of whether or not variations in albedo observed in our sample (Table 2) are indicative of dynamical family membership instead. Utilizing the Hierarchical Clustering Method (Zappalà et al. 1990) which utilizes proper elements of asteroids and searches phase space for clusters of objects with similar velocities, Broz \& Vokrouhlicky (2008) identify two distinct dynamical families within the Hilda obital group. Of these two dynamical families, Hilda and Schubart, identified by Broz \& Vokrouhlicky (2008), only the Schubart dynamical family contributes significantly to our Hilda asteroid group sample. The 16 members of the Schubart family present in our sample follow the same albedo trend as seen within the rest of the Hilda asteroid group as illustrated in Fig. 3. Thus dynamical family membership cannot be delineated solely by albedo.

Alternatively, the albedo-diameter relation may instead be indicative of the influence of collisional processes within Hilda asteroid group. With increased age and thus increased ion irradiation exposure, surfaces with organic compositions redden and the geometric albedo is reduced (Andronico et al. 1987; Moroz et al. 2004). This is the reported cause for the albedo-diameter relation for small Trojan asteroids (Fernandez et al. 2009). Evidence of the space weathering reddening effect is observed for Hildas in the optical (Dahlgren \& Lagerkvist 1995), where a trend towards D-type asteroids is apparent with decreasing diameter for asteroids with $\mathrm{D}>20 \mathrm{~km}$. It is however problematic to infer a 
connection between albedo and the potential for space weathering for small diameter objects as the small Hilda asteroids seen in Gil-Hutton \& Brunini (2008) display a significant range of spectral slopes and thus taxonomic types in the range $12<\mathrm{H}<16$. This spectral range is characteristic of the C-, D-, and X- taxonomic types as derived by Bus \& Binzel (2002) and the range of small Hilda group asteroid albedos corresponds with the range in albedos for these taxonomic types as derived in Ryan \& Woodward (2010). If the albedo-diameter relation is indicative of space weathering and subsequent reddening and reduced albedo with continued exposure to solar flux, one would also expect that a clear trend of increasing spectral slope as an inverse function of absolute magnitude, which is not apparent from the work of Gil-Hutton \& Brunini (2008).

Interestingly, the albedo distribution of small Hilda asteroids is quite similar to the albedo distribution of cold classical KBOs (Brucker et al. 2009). Cold classical KBOs are thought to be the original proto-Kuiper Belt population (Levison et al. 2008), suggesting that the Hilda asteroid group may be contaminated by bodies which originated in orbits past Uranus and Neptune. The dynamical models of Levison et al. (2009), predict that $\sim 8 \%$ of the Hilda population can be populated by bodies which originated in the Kuiper Belt and were subsequently transported into the inner solar system via giant planet migration. Our Hilda asteroid group sample contains 6 asteroids with $p_{V}>0.1$, an albedo range characteristic of cold classical KBOs and higher than the albedo range for standard D- and C-type asteroids. Furthermore, number counts derived from the $24 \mu \mathrm{m}$ MIPS mid-IR fluxes above the $5 \sigma$ detection threshold imply that up to $11 \%$ of the small Hilda asteroid group population is contaminated with objects that may have originated within the Kuiper Belt. 


\section{CONCLUSIONS}

We have measured the $24 \mu \mathrm{m}$ thermal emission from 62 small Hilda group asteroids obtained by Spitzer MIPS instrument and have combined this archival data set with $\mathrm{H}$ magnitudes to calculate effective diameters and albedos. Our object sample spans a range of diameters from 3 to $12 \mathrm{~km}$ which is significantly smaller than the D > $30 \mathrm{~km}$ Hilda group asteroids for which albedos and diameters are available from IRAS observations Ryan \& Woodward (2010). Based on our analysis of the MIPS photometry, we conclude:

The measured mean albedo of our small Hilda asteroid sample is $p_{V}=0.07 \pm 0.05$. This albedo is higher than the mean albedo of large Hildas which is found to be $p_{V}=0.04$ \pm 0.01 by Ryan \& Woodward (2010) and the small Hilda asteroids exhibit greater albedo diversity than the larger members of the same dynamical population.

The geometric albedo increases with decreasing diameter for Hildas with diameters in the 4 to $12 \mathrm{~km}$ range. The correlation is significant to a $5.97 \sigma$ and addition of large Hilda asteroids from IRAS observations (Ryan \& Woodward 2010) increases the significance of this correlation to $6.3 \sigma$. Though this trend could be considered to be a result of collisional processes and a tracer of space weathering, the spectral diversity of the Hilda asteroid group complicates this interpretation and colors and/or taxonomic determinations for objects in this sample are required before any firm statements can be made regarding a collisional processing and space weathering link.

The power-law slope of the Hilda asteroid group size-frequency distribution breaks at $\simeq 12.5 \mathrm{~km}$ when the albedo-diameter relation for Hilda asteroids is applied. This break is found to not be an observational bias in optical surveys, but rather a real signature wherein only $\sim 30 \%$ of asteroids with $5 \mathrm{~km}$ diameters predicted from the size frequency distribution above $15 \mathrm{~km}$ are observed. Given the low collisional probabilities within the Hilda asteroid group as a whole (Dell'Oro et al. 2001), it is unlikely that this depletion is due strictly to 
Hilda-Hilda collisions resulting in small fragments with velocities sufficient to escape the resonance and is more likely the result of early depletion of the small Hilda population such as a period of intense cometary bombardment as suggested by Gil-Hutton \& Brunini (2000) or bombardment from Kuiper Belt planetesimals as suggested by Levison et al. (2008). As $\sim 10 \%$ of our Hilda asteroid group sample contains asteroids with albedos commensurate with the albedos of cold classical Kuiper Belt objects, searches for Kuiper Belt contaminants within the outer solar system should include taxonomic classification and spectroscopic follow up of these asteroid targets.

E.R. and C.E.W. acknowledge support from the National Science Foundation grant AST-0706980 to conduct this research.

The authors thank the efforts of an anonymous referee, whose suggestions improved our manuscript. Facilities: Spitzer 


\section{REFERENCES}

Andronico, G., Baratta, G. A., Spinella, F., \& Strazzulla, G. 1987, A\&A, 184, 333

Broz, M. \& Vokrouhlicky, D. 2008, MNRAS, 390, 715

Brucker, M. J, Grundy, W. M., Stansberry, J. A., Spencer, J. R., Sheppard, S. S., Chiang, E. I., \& Buie, M. W. 2009, Icarus, 201, 284.

Bus, S.J., Binzel, R.P. 2002 Icarus, 158, 106

Dell'Oro,A., Marzari, F., Paolicchi, P., Vanzani, V. 2001, A\&A, 366, 1053

Dahlgren, M. \& Lagerkvist, C. 1995, A\&A, 302, 907

Dahlgren, M., Lahulla, J. F., Lagerkvist, C.-I., Lagerros, J., Mottola, S., Erickson, A., Gonano-Beurer, M., \& DiMartino, M., 1998, Icarus, 133, 247

Delbo, M., et al., 2007, Icarus, 190, 236

Delbó, M., \& Tanga, P. 2009, Planetary Space Sci., 57, 259

Dohnanyi, J. W. 1969, J. Geophys. Res., 74, 2531

Engelbracht, C. W., et al. 2007, PASP, 119, 999

Fernandez, Y.R, et al. 2008, in Asteroids, Comets,Meteors 2008, LPI Contrib. 1405, (Houston, TX: Lunar and Planetary Institute), 8307

Fernandez, Y.R, Jewitt, D., Ziffer, J.E 2009, AJ, 138, 240

Franklin, F., et al. 2004, AJ, 128, 1391

Gil-Hutton, R. \& Brunini, A. 2008, Icarus, 193, 567

Gil-Hutton, R. \& Brunini, A. 2000, Icarus, 145, 382 
Gladman, B. J., et al. 2009, Icarus, 202, 104

Gordon, K. D., et al. 2005, PASP, 117, 503

Gradie, J. C., Chapman, C. R., Tedesco, E. F. 1989, in Asteroids II, eds. R. P. Binzel, T. Gehrels, and M. S. Mathews, (U. Arizona Press: Tucson), p. 316

Grimm, R.E. and McSween, H.Y. Jr, 1993, Science, 259, 653

Gulbis, A. A. S, Elliot, J. L., Kane, J. F. 2006, Icarus, 183, 168

Hainaut, O. R. \& Delsanti, A. C. 2002, A\&A, 389, 641

Harris, A. W. 1998, Icarus, 131, 291

Hsieh, H., Jewitt, D., \& Fernández, Y. R. 2009, ApJ, 694. L111

Ivezić, Z̆., et al. 2001, AJ, 122, 2749

Lamy, P., Toth, I., Fernández, Y. R., \& Weaver, H. A., 2004, in Comets II, eds. M. Festou, H. U. Keller, H. A. Weaver, (Univ. Arizona Press: Tucson), p. 223

Lamy, P. \& Toth, I., 2009, Icarus, 201, 674

Larsen, J. A., et al. 2007, AJ, 133, 1247

Lebofsky, L. A. \& Spencer, J. 1989, in Asteroids II, eds. R. P. Binzel, T. Gehrels, and M. S. Matthews, (Univ. Arizona Press: Tucson), p. 128

Levison, H. F., Morbidelli, A., Van Laerhoven, C., Gomes, R., Tsiganis, K. 2008, Icarus, 196,258

Levison, H. F, Bottke, W. F., Gounelle, M., Morbidelli, A., Nesvorny, D., Tsiganis, K. 2009, Nature, 460, 364 
Makovoz, D. \& Marleau, F. R. 2005, PASP, 117, 1113

McCoy, T. J., Mittlefehldt, D. W., \& Wilson, L. 2010, in Meteorites and the Early Solar System II, eds. D. S. Lauretta and H. Y. McSweeney, Jr. (U. Arizona Press: Tucson), p.733

Meyers, J. L., \& Well, A. D. 2003, Research Design and Statistical Analysis, eds. L. Erlbaum, $2^{\text {nd }}$ edition, p.508 isbn 0805840370

Morrison, D., 1973, Icarus, 19, 1

Moroz, L., Baratta, G., Strazzulla, G., Starukhina, L., Dotto, E., Barucci, M.A., Arnold, G., \& Distefano, E. 2004, Icarus, 170, 214

Rieke, G. H., et al. 2004, ApJS, 154, 25

Ryan, E. L., \& Woodward, C. E. 2010, AJ, 140, 933

Stansberry, J.A, et al., 2007, PASP, 119,1038

Wolters, S. D., et al. 2008, Icarus, 193, 535

Werner, M. W., et al. 2004, ApJS, 154, 1

Zappalà, V., Cellino, A., Farinella, P., Knežević, Z. 1990, AJ, 100, 2030 
Table 1. Orbital elements and $24 \mu \mathrm{m}$ fluxes for selected Hildas

\begin{tabular}{|c|c|c|c|c|c|c|c|c|c|c|c|}
\hline Number & $\begin{array}{c}\text { Name/Provisional } \\
\text { Designation }\end{array}$ & $\begin{array}{c}\text { Dynamical } \\
\text { Family } \\
\text { flag }\end{array}$ & $\begin{array}{l}\text { Request } \\
\text { Key }\end{array}$ & $\begin{array}{c}\text { UT } \\
\text { Date } \\
\text { (yyyy-mm-dd) }\end{array}$ & $\begin{array}{c}\text { UT } \\
\text { Time } \\
\text { (at start) }\end{array}$ & $\begin{array}{l}\text { Heliocentric } \\
\text { Distance } \\
\text { (AU) }\end{array}$ & $\begin{array}{c}\text { Geocentric } \\
\text { Distance } \\
(\mathrm{AU})\end{array}$ & $\begin{array}{l}\text { Phase } \\
\text { Angle } \\
(\operatorname{deg})\end{array}$ & $\begin{array}{l}\text { Absolute } \\
\text { Magnitude }\end{array}$ & $\begin{array}{l}24 \mu \mathrm{m} \\
\text { Flux } \\
(\mu \mathrm{Jy})\end{array}$ & $\begin{array}{l}\text { Flux } \\
\text { Error } \\
(\mu \mathrm{Jy})\end{array}$ \\
\hline 21804 & Vaclavneumann & G & 23241472 & 2008-02-16 & $12: 54: 14$ & 3.24 & 2.75 & 16.91 & 14.500 & 79050.01 & 1588.5 \\
\hline 55347 & 2001SH142 & G & 23241728 & $2008-06-27$ & 13:08:09 & 3.74 & 3.33 & 15.08 & 14.500 & 36310.0 & 738.8 \\
\hline 79097 & $1981 \mathrm{EC} 24$ & $\mathrm{~s}$ & 23241984 & 2007-11-29 & 15:33:04 & 4.29 & 3.69 & 11.89 & 14.500 & 25920.0 & 534.0 \\
\hline 89903 & Post & G & 23242240 & 2008-02-16 & $12: 45: 02$ & 3.46 & 3.16 & 16.64 & 14.500 & 10710.0 & 252.7 \\
\hline 90502 & Burrati & $\mathrm{H}$ & 23242496 & $2007-11-28$ & $22: 51: 12$ & 4.69 & 4.44 & 12.41 & 14.500 & 10910.0 & 253.0 \\
\hline 99862 & Kenlevin & $\mathrm{H}$ & 23242752 & 2008-04-15 & $16: 26: 49$ & 4.79 & 4.37 & 11.43 & 14.500 & 6570.0 & 180.0 \\
\hline 104876 & 2000HН98 & G & 23243008 & 2008-03-14 & $03: 46: 54$ & 4.22 & 4.02 & 13.69 & 14.500 & 3956.0 & 157.3 \\
\hline 120175 & 2003KB11 & G & 23243264 & 2007-11-30 & $05: 24: 38$ & 4.97 & 4.59 & 11.37 & 14.500 & 5184.0 & 162.4 \\
\hline 131502 & $2001 \mathrm{SW} 273$ & $\mathrm{~s}$ & 23243520 & 2007-09-26 & $01: 45: 52$ & 3.29 & 3.02 & 18.04 & 14.500 & 50340.01 & 1018.4 \\
\hline 136835 & 1997UL19 & G & 23243776 & 2008-02-16 & 21:08:06 & 3.61 & 3.35 & 16.07 & 14.500 & 22350.0 & 468.4 \\
\hline 136935 & 1998QK6 & G & 23244288 & 2007-11-30 & 05:41:12 & 4.04 & 3.64 & 13.99 & 13.900 & 19940.0 & 419.1 \\
\hline 39382 & Opportunity & G & 23244544 & $2007-08-23$ & 22:04:39 & 3.44 & 3.12 & 17.11 & 14.600 & 22250.0 & 466.8 \\
\hline 58353 & 1995EW4 & G & 23244800 & 2008-01-04 & $02: 10: 45$ & 3.56 & 3.46 & 16.48 & 14.600 & 33170.0 & 680.8 \\
\hline 65989 & 1998KZ12 & G & 23245056 & $2007-10-27$ & 11:13:43 & 3.93 & 3.52 & 14.49 & 14.600 & 29410.0 & 604.6 \\
\hline 128254 & 2003SL259 & $\mathrm{s}$ & 23245312 & 2008-03-17 & 08:11:46 & 4.96 & 4.88 & 11.67 & 14.600 & 5080.0 & 173.0 \\
\hline 128858 & $2004 \mathrm{SQ} 20$ & $\mathrm{~s}$ & 23245568 & 2008-03-14 & $11: 55: 38$ & 4.60 & 4.53 & 12.58 & 14.600 & 10040.0 & 247.1 \\
\hline 134429 & 1998RT5 & $\mathrm{S}$ & 23245824 & 2007-11-30 & 05:16:50 & 3.81 & 3.41 & 14.96 & 14.500 & 29390.0 & 603.8 \\
\hline 134562 & 1999RS177 & $\mathrm{s}$ & 23246088 & $2007-08-23$ & $23: 06: 59$ & 3.14 & 2.67 & 18.08 & 13.700 & 65200.01 & 1313.4 \\
\hline 145396 & 2005NE53 & G & 23246336 & 2008-03-13 & $18: 14: 29$ & 4.35 & 3.76 & 11.48 & 14.600 & 4661.0 & 150.4 \\
\hline 145718 & 1993FT57 & $\mathrm{S}$ & 23246592 & 2008-05-16 & $12: 48: 45$ & 3.45 & 2.88 & 15.10 & 14.600 & 28820.0 & 590.7 \\
\hline 145960 & $1999 X V 255$ & G & 23246848 & $2007-11-29$ & $10: 38: 27$ & 3.64 & 3.41 & 16.18 & 14.600 & 21480.0 & 452.1 \\
\hline 62959 & 2000VV39 & G & 23247104 & $2007-11-28$ & $22: 42: 01$ & 3.60 & 2.99 & 14.23 & 14.700 & 19930.0 & 417.7 \\
\hline 83877 & 2001UE96 & G & 23247360 & $2007-10-27$ & 08:22:56 & 4.67 & 4.07 & 10.94 & 14.700 & 9674.0 & 229.3 \\
\hline 116489 & $2004 \mathrm{BN} 12$ & G & 23247616 & $2007-10-27$ & 11:05:38 & 4.14 & 3.75 & 13.80 & 14.700 & 14440.0 & 316.7 \\
\hline 116512 & 2004BN38 & G & 23247872 & $2007-10-27$ & $11: 21: 22$ & 4.44 & 4.04 & 12.74 & 14.700 & 5759.0 & 172.2 \\
\hline 117667 & $2005 \mathrm{EC} 205$ & G & 23248128 & $2007-10-28$ & $06: 46: 22$ & 4.43 & 4.09 & 13.04 & 14.700 & 1967.0 & 143.5 \\
\hline 118177 & $1992 \mathrm{EZ} 13$ & G & 23248384 & $2008-02-17$ & $12: 20: 07$ & 3.65 & 3.15 & 14.81 & 14.300 & 54210.01 & 1093.3 \\
\hline 120761 & $1998 \mathrm{AX} 1$ & $\mathrm{~s}$ & 23248640 & 2008-03-14 & $14: 37: 51$ & 4.55 & 4.57 & 12.63 & 14.700 & 8685.0 & 228.9 \\
\hline 125130 & 2001UO56 & G & 23248896 & $2008-06-27$ & $13: 16: 37$ & 3.28 & 2.92 & 17.61 & 14.100 & 19700.0 & 415.2 \\
\hline 128295 & 2003WD111 & G & 23249152 & 2008-04-15 & $17: 16: 58$ & 4.46 & 4.10 & 12.57 & 14.700 & 1764.0 & 128.9 \\
\hline 129241 & $2005 \mathrm{QS} 13$ & $\mathrm{~s}$ & 23249408 & 2007-11-29 & 10:29:11 & 4.54 & 4.12 & 12.31 & 14.700 & 12200.0 & 276.0 \\
\hline 129634 & $1998 \mathrm{HP} 43$ & G & 23249664 & $2007-10-27$ & $11: 29: 26$ & 3.80 & 3.36 & 14.84 & 14.000 & 22090.0 & 461.1 \\
\hline 131481 & 2001RT111 & G & 23249920 & $2007-08-27$ & $11: 27: 31$ & 3.91 & 3.65 & 15.10 & 14.600 & 12330.0 & 281.6 \\
\hline
\end{tabular}


Table 1-Continued

\begin{tabular}{|c|c|c|c|c|c|c|c|c|c|c|c|}
\hline Number & $\begin{array}{c}\text { Name/Provisional } \\
\text { Designation }\end{array}$ & $\begin{array}{c}\text { Dynamical } \\
\text { Family } \\
\text { flag }\end{array}$ & $\begin{array}{c}\text { Request } \\
\text { Key }\end{array}$ & $\begin{array}{c}\text { UT } \\
\text { Date } \\
\text { (yyyy-mm-dd) }\end{array}$ & $\begin{array}{c}\text { UT } \\
\text { Time } \\
\text { (at start) }\end{array}$ & $\begin{array}{l}\text { Heliocentric } \\
\text { Distance } \\
(\mathrm{AU})\end{array}$ & $\begin{array}{l}\text { Geocentric } \\
\text { Distance } \\
\text { (AU) }\end{array}$ & $\begin{array}{l}\text { Phase } \\
\text { Angle } \\
\text { (deg) }\end{array}$ & $\begin{array}{c}\text { Absolute } \\
\text { Magnitude }\end{array}$ & $\begin{array}{c}24 \mu \mathrm{m} \\
\text { Flux } \\
(\mu \mathrm{Jy})\end{array}$ & $\begin{array}{l}\text { Flux } \\
\text { Error } \\
(\mu \mathrm{Jy})\end{array}$ \\
\hline 145368 & $2005 \mathrm{MB} 43$ & G & 23250176 & $2007-11-30$ & $10: 14: 48$ & 3.99 & 3.54 & 13.94 & 14.700 & 16820.0 & 360.3 \\
\hline 63491 & 2001 OY 60 & $\mathrm{~s}$ & 23250432 & 2008-04-15 & $17: 08: 10$ & 3.73 & 3.46 & 15.44 & 14.800 & 36650.0 & 747.6 \\
\hline 64823 & 2001 XO240 & G & 23250688 & 2008-08-30 & $20: 35: 04$ & 3.35 & 3.33 & 17.65 & 14.800 & 12540.0 & 291.7 \\
\hline 73769 & 1994PN12 & $\mathrm{s}$ & 23250944 & 2008-03-14 & $12: 10: 18$ & 4.67 & 4.59 & 12.41 & 14.800 & 2657.0 & 149.7 \\
\hline 79096 & 1981EM20 & $\mathrm{H}$ & 23251200 & 2008-07-29 & $12: 50: 03$ & 4.49 & 4.21 & 12.95 & 14.800 & 13780.0 & 306.9 \\
\hline 85142 & 1981EO29 & G & 23251456 & $2007-11-28$ & $06: 58: 57$ & 4.44 & 3.98 & 12.41 & 14.800 & 13830.0 & 302.7 \\
\hline 119904 & $2002 \mathrm{EX} 6$ & G & 23251712 & $2007-11-29$ & $12: 20: 27$ & 4.62 & 4.21 & 12.14 & 14.100 & 10500.0 & 243.9 \\
\hline 129002 & 2004TR256 & $\mathrm{H}$ & 23251968 & 2008-04-15 & $17: 25: 32$ & 4.50 & 4.04 & 12.03 & 14.800 & 5839.0 & 168.2 \\
\hline 133559 & 2003UZ10 & G & 23252224 & 2008-04-15 & $16: 44: 22$ & 5.05 & 4.72 & 11.17 & 14.800 & 2364.0 & 137.4 \\
\hline 134233 & 2005YD54 & G & 23252480 & 2008-03-14 & 03:24:36 & 4.48 & 4.19 & 12.73 & 14.800 & 4226.0 & 152.6 \\
\hline 141518 & $2002 \mathrm{~EB} 136$ & $\mathrm{H}$ & 23252736 & 2007-11-29 & $12: 02: 25$ & 4.35 & 3.79 & 12.00 & 14.100 & 21750.0 & 452.6 \\
\hline 141701 & $2002 \mathrm{KM} 15$ & $\mathrm{~S}$ & 23252992 & $2007-11-29$ & $21: 15: 12$ & 4.16 & 4.00 & 14.15 & 14.800 & 6767.0 & 191.9 \\
\hline 145397 & 2005 NC5 4 & G & 23253248 & 2008-03-13 & $10: 52: 27$ & 4.37 & 3.80 & 11.66 & 14.800 & 6766.0 & 180.9 \\
\hline 145421 & 2005PD19 & $\mathrm{s}$ & 23253504 & 2007-11-29 & $21: 24: 34$ & 4.31 & 3.95 & 13.27 & 14.800 & 13400.0 & 298.7 \\
\hline 52079 & 2002RU61 & G & 23253760 & 2008-03-14 & 03:56:04 & 4.15 & 4.15 & 13.90 & 14.900 & 17270.0 & 376.6 \\
\hline 62489 & $2000 \mathrm{SS} 223$ & $\mathrm{~s}$ & 23254016 & $2007-11-28$ & $17: 01: 12$ & 4.09 & 3.57 & 13.16 & 14.900 & 27430.0 & 564.7 \\
\hline 127519 & $2002 \mathrm{UJ} 16$ & G & 23254272 & 2008-04-15 & $16: 35: 41$ & 3.86 & 3.52 & 14.76 & 14.300 & 18490.0 & 393.0 \\
\hline 143658 & $2003 \mathrm{SB} 45$ & G & 23254528 & 2008-04-15 & $16: 52: 11$ & 4.94 & 4.63 & 11.48 & 14.900 & 1114.0 & 130.9 \\
\hline 145373 & 2005MV49 & G & 23254784 & $2007-11-30$ & $10: 23: 43$ & 3.76 & 3.43 & 15.42 & 14.900 & 14100.0 & 312.2 \\
\hline 133324 & 2003SY90 & G & 23255040 & 2008-03-14 & 04:04:36 & 4.77 & 4.71 & 12.12 & 15.000 & 6020.0 & 186.2 \\
\hline 145767 & $1997 \mathrm{PW}$ & G & 23255296 & $2007-11-30$ & $05: 32: 48$ & 4.68 & 4.25 & 11.92 & 15.000 & 3756.0 & 145.8 \\
\hline 134016 & 2004 VU 53 & G & 23255552 & 2008-04-15 & $17: 00: 16$ & 4.94 & 4.68 & 11.56 & 15.100 & 2942.0 & 143.6 \\
\hline 134690 & 1999XP61 & G & 23255808 & 2008-03-13 & 10:31:16 & 3.48 & 2.96 & 15.37 & 14.300 & 48220.0 & 974.4 \\
\hline 143621 & 2003GE55 & G & 23256064 & 2007-11-29 & 12:11:07 & 4.72 & 4.15 & 10.95 & 14.500 & 11720.0 & 265.6 \\
\hline 64390 & 2001UY149 & G & 23256320 & $2007-11-28$ & 23:00:48 & 4.39 & 4.15 & 13.32 & 15.200 & 9007.0 & 225.1 \\
\hline 145841 & 1998YH20 & G & 23256576 & $2008-02-17$ & $12: 10: 41$ & 3.83 & 3.57 & 15.09 & 15.000 & 15470.0 & 337.2 \\
\hline 129007 & 2004TP296 & G & 23256832 & 2008-03-14 & 03:38:42 & 4.99 & 4.83 & 11.57 & 15.500 & 3147.0 & 149.9 \\
\hline 142470 & $2002 \mathrm{TE} 13$ & G & 23257088 & $2007-08-22$ & $12: 28: 12$ & 4.54 & 4.49 & 13.01 & 14.900 & 7620.0 & 209.0 \\
\hline 147836 & $2005 \mathrm{TN} 125$ & $\mathrm{~S}$ & 23244032 & 2009-03-23 & $10: 59: 29$ & 3.59 & 3.43 & 16.21 & 14.500 & 30580.0 & 629.0 \\
\hline
\end{tabular}


Note. - In column 3 the flags are the following: $\mathrm{H}=$ member of the Hilda dynamical family as determined by Broz \& Vokrouhlicky (2008), S = member of the Shubart dynamical family as determined by Broz \& Vokrouhlicky (2008) and G= member of Hilda group without either Hilda or Schubart family correspondence 
Table 2. MIPS $24 \mu \mathrm{m}$ HILDA ASTEROID GROUP THERMAL MODEL SOLUTIONS

\begin{tabular}{lcccc}
\hline \hline \multirow{1}{*}{ Name } & STM & STM & NEATM & NEATM \\
& Albedo & Diameter & Albedo & Diameter \\
& & $(\mathrm{km})$ & & $(\mathrm{km})$ \\
\hline Vaclavneumann & $0.025 \pm 0.000$ & $10.59 \pm 0.06$ & $0.024 \pm 0.000$ & $10.73 \pm 0.06$ \\
2001SH142 & $0.061 \pm 0.001$ & $9.61 \pm 0.05$ & $0.058 \pm 0.001$ & $9.81 \pm 0.06$ \\
1981EC25 & $0.024 \pm 0.000$ & $11.35 \pm 0.07$ & $0.023 \pm 0.001$ & $11.64 \pm 0.28$ \\
Post & $0.191 \pm 0.003$ & $4.76 \pm 0.03$ & $0.185 \pm 0.003$ & $4.84 \pm 0.03$ \\
Burrati & $0.090 \pm 0.001$ & $7.94 \pm 0.05$ & $0.084 \pm 0.001$ & $8.22 \pm 0.05$ \\
Kenlevin & $0.079 \pm 0.001$ & $6.41 \pm 0.03$ & $0.073 \pm 0.001$ & $6.66 \pm 0.05$ \\
2000HH98 & $0.181 \pm 0.003$ & $4.24 \pm 0.04$ & $0.171 \pm 0.003$ & $4.36 \pm 0.03$ \\
2003KB11 & $0.081 \pm 0.001$ & $6.10 \pm 0.04$ & $0.075 \pm 0.001$ & $6.34 \pm 0.04$ \\
2001SW273 & $0.039 \pm 0.000$ & $9.37 \pm 0.06$ & $0.038 \pm 0.000$ & $9.48 \pm 0.06$ \\
1997UL19 & $0.052 \pm 0.001$ & $7.38 \pm 0.05$ & $0.050 \pm 0.001$ & $7.51 \pm 0.04$ \\
1998QK6 & $0.042 \pm 0.001$ & $8.13 \pm 0.06$ & $0.040 \pm 0.001$ & $8.35 \pm 0.05$ \\
Opportunity & $0.058 \pm 0.001$ & $6.66 \pm 0.04$ & $0.056 \pm 0.001$ & $6.75 \pm 0.04$ \\
1995EW4 & $0.048 \pm 0.001$ & $9.20 \pm 0.07$ & $0.046 \pm 0.001$ & $9.35 \pm 0.06$ \\
1998KZ12 & $0.092 \pm 0.001$ & $9.46 \pm 0.06$ & $0.088 \pm 0.001$ & $9.69 \pm 0.06$ \\
2003SL259 & $0.067 \pm 0.001$ & $6.45 \pm 0.05$ & $0.062 \pm 0.001$ & $6.70 \pm 0.06$ \\
1998RT5 & $0.032 \pm 0.000$ & $8.91 \pm 0.05$ & $0.031 \pm 0.000$ & $9.10 \pm 0.07$ \\
2005NE9820 & $0.040 \pm 0.000$ & $7.91 \pm 0.04$ & $0.038 \pm 0.000$ & $8.18 \pm 0.05$ \\
& $0.125 \pm 0.002$ & $4.36 \pm 0.04$ & $0.117 \pm 0.001$ & $4.51 \pm 0.03$
\end{tabular}


Table 2-Continued

\begin{tabular}{lcccc}
\hline \hline \multirow{2}{*}{ Name } & STM & STM & NEATM & NEATM \\
& Albedo & Diameter & Albedo & Diameter \\
& & $(\mathrm{km})$ & & $(\mathrm{km})$ \\
\hline 1993FT57 & $0.054 \pm 0.001$ & $6.93 \pm 0.04$ & $0.052 \pm 0.001$ & $7.06 \pm 0.04$ \\
1999XV255 & $0.052 \pm 0.001$ & $7.41 \pm 0.05$ & $0.050 \pm 0.001$ & $7.55 \pm 0.05$ \\
2000VV39 & $0.114 \pm 0.001$ & $6.19 \pm 0.04$ & $0.109 \pm 0.001$ & $6.33 \pm 0.04$ \\
2001UE96 & $0.091 \pm 0.001$ & $7.15 \pm 0.04$ & $0.084 \pm 0.001$ & $7.43 \pm 0.05$ \\
2004BN12 & $0.067 \pm 0.001$ & $7.31 \pm 0.05$ & $0.064 \pm 0.001$ & $7.52 \pm 0.04$ \\
2004BN38 & $0.111 \pm 0.001$ & $5.21 \pm 0.03$ & $0.104 \pm 0.001$ & $5.38 \pm 0.03$ \\
2005EC205 & $0.257 \pm 0.007$ & $3.16 \pm 0.04$ & $0.242 \pm 0.006$ & $3.25 \pm 0.04$ \\
1992EZ13 & $0.029 \pm 0.000$ & $10.86 \pm 0.08$ & $0.027 \pm 0.001$ & $11.13 \pm 0.24$ \\
1998AX1 & $0.046 \pm 0.001$ & $7.33 \pm 0.06$ & $0.043 \pm 0.001$ & $7.57 \pm 0.05$ \\
2001UO56 & $0.072 \pm 0.001$ & $5.68 \pm 0.03$ & $0.070 \pm 0.001$ & $5.76 \pm 0.03$ \\
2003WD111 & $0.240 \pm 0.005$ & $3.25 \pm 0.03$ & $0.225 \pm 0.004$ & $3.35 \pm 0.03$ \\
2005QS13 & $0.042 \pm 0.001$ & $7.86 \pm 0.05$ & $0.040 \pm 0.000$ & $8.13 \pm 0.05$ \\
1998HP43 & $0.041 \pm 0.001$ & $7.58 \pm 0.05$ & $0.039 \pm 0.000$ & $7.75 \pm 0.05$ \\
2001RT111 & $0.059 \pm 0.001$ & $6.29 \pm 0.04$ & $0.056 \pm 0.001$ & $6.43 \pm 0.03$ \\
2005MB43 & $0.057 \pm 0.001$ & $7.20 \pm 0.04$ & $0.054 \pm 0.001$ & $7.39 \pm 0.04$ \\
2001OY60 & $0.039 \pm 0.000$ & $10.00 \pm 0.06$ & $0.038 \pm 0.000$ & $10.19 \pm 0.06$ \\
2001XO240 & $0.137 \pm 0.002$ & $5.32 \pm 0.03$ & $0.133 \pm 0.002$ & $5.39 \pm 0.03$ \\
1994PN12 & $0.153 \pm 0.003$ & $4.26 \pm 0.04$ & $0.143 \pm 0.003$ & $4.41 \pm 0.05$ \\
1981EM20 & $0.054 \pm 0.001$ & $8.51 \pm 0.06$ & $0.051 \pm 0.001$ & $8.79 \pm 0.06$
\end{tabular}


Table 2-Continued

\begin{tabular}{lcccc}
\hline \hline \multirow{2}{*}{ Name } & STM & STM & NEATM & NEATM \\
& Albedo & Diameter & Albedo & Diameter \\
& & $(\mathrm{km})$ & & $(\mathrm{km})$ \\
\hline & & & & \\
1981EO29 & $0.057 \pm 0.001$ & $8.07 \pm 0.04$ & $0.054 \pm 0.001$ & $8.33 \pm 0.05$ \\
2002EX6 & $0.037 \pm 0.000$ & $7.57 \pm 0.04$ & $0.034 \pm 0.000$ & $7.85 \pm 0.04$ \\
2004TR256 & $0.083 \pm 0.001$ & $5.33 \pm 0.04$ & $0.078 \pm 0.001$ & $5.52 \pm 0.04$ \\
2003UZ10 & $0.110 \pm 0.003$ & $4.43 \pm 0.05$ & $0.102 \pm 0.002$ & $4.62 \pm 0.05$ \\
2005YD54 & $0.106 \pm 0.002$ & $4.71 \pm 0.04$ & $0.100 \pm 0.001$ & $4.87 \pm 0.04$ \\
2002EB136 & $0.024 \pm 0.000$ & $9.37 \pm 0.07$ & $0.023 \pm 0.000$ & $9.59 \pm 0.03$ \\
2002KM15 & $0.081 \pm 0.001$ & $5.38 \pm 0.04$ & $0.077 \pm 0.001$ & $5.53 \pm 0.04$ \\
2005NC54 & $0.074 \pm 0.001$ & $5.25 \pm 0.03$ & $0.069 \pm 0.001$ & $5.43 \pm 0.03$ \\
2005PD19 & $0.045 \pm 0.000$ & $7.61 \pm 0.04$ & $0.043 \pm 0.001$ & $7.84 \pm 0.05$ \\
2002RU61 & $0.053 \pm 0.001$ & $8.86 \pm 0.05$ & $0.050 \pm 0.001$ & $9.11 \pm 0.05$ \\
2000SS223 & $0.058 \pm 0.001$ & $9.48 \pm 0.06$ & $0.055 \pm 0.001$ & $9.74 \pm 0.07$ \\
2002UJ16 & $0.036 \pm 0.000$ & $7.37 \pm 0.05$ & $0.034 \pm 0.000$ & $7.54 \pm 0.05$ \\
2003SB45 & $0.316 \pm 0.013$ & $2.89 \pm 0.06$ & $0.294 \pm 0.013$ & $2.99 \pm 0.07$ \\
2005MV49 & $0.051 \pm 0.001$ & $6.12 \pm 0.04$ & $0.049 \pm 0.001$ & $6.24 \pm 0.04$ \\
2003SY90 & $0.059 \pm 0.001$ & $6.59 \pm 0.04$ & $0.055 \pm 0.001$ & $6.83 \pm 0.05$ \\
1997PW & $0.075 \pm 0.001$ & $4.54 \pm 0.04$ & $0.070 \pm 0.001$ & $4.69 \pm 0.03$ \\
2004VU53 & $0.079 \pm 0.002$ & $4.66 \pm 0.04$ & $0.073 \pm 0.001$ & $4.84 \pm 0.04$ \\
1999XP61 & $0.039 \pm 0.000$ & $9.30 \pm 0.04$ & $0.038 \pm 0.000$ & $9.47 \pm 0.05$ \\
2003GE55 & $0.025 \pm 0.000$ & $7.96 \pm 0.05$ & $0.023 \pm 0.001$ & $8.34 \pm 0.24$
\end{tabular}


Table 2-Continued

\begin{tabular}{ccccc}
\hline \hline \multirow{2}{*}{ Name } & STM & STM & NEATM & NEATM \\
& Albedo & Diameter & Albedo & Diameter \\
& & $(\mathrm{km})$ & & $(\mathrm{km})$ \\
\hline & & & & \\
2001UY149 & $0.071 \pm 0.001$ & $6.62 \pm 0.04$ & $0.067 \pm 0.001$ & $6.82 \pm 0.04$ \\
1998YH20 & $0.032 \pm 0.000$ & $6.75 \pm 0.03$ & $0.031 \pm 0.000$ & $6.90 \pm 0.04$ \\
2004TP296 & $0.062 \pm 0.001$ & $5.06 \pm 0.05$ & $0.057 \pm 0.001$ & $5.26 \pm 0.05$ \\
2002 TE13 & $0.024 \pm 0.000$ & $6.75 \pm 0.05$ & $0.023 \pm 0.001$ & $6.95 \pm 0.11$ \\
$2005 T N 125$ & $0.042 \pm 0.001$ & $8.93 \pm 0.06$ & $0.041 \pm 0.000$ & $9.10 \pm 0.05$ \\
\hline
\end{tabular}



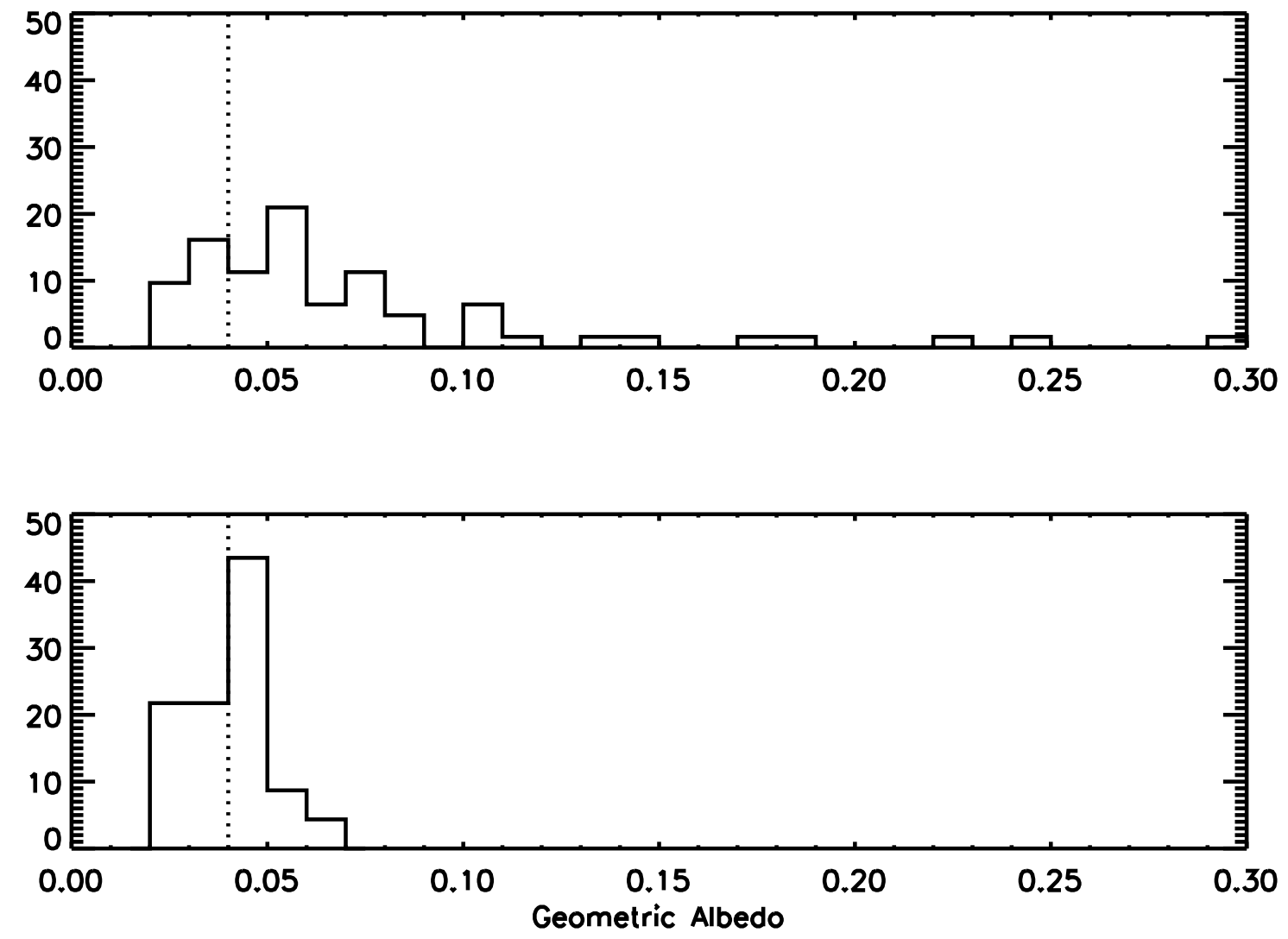

Fig. 1. - Histogram plots of IRAS albedos (derived from Ryan \& Woodward 2010) from NEATM (bottom panel) and Spitzer albedos from NEATM (top panel) normalized such that the sum of all objects in each sample equals 100.The dashed line indicates the mean albedo of the Hilda asteroids detected with IRAS (Rvan \& Woodward 2010). 


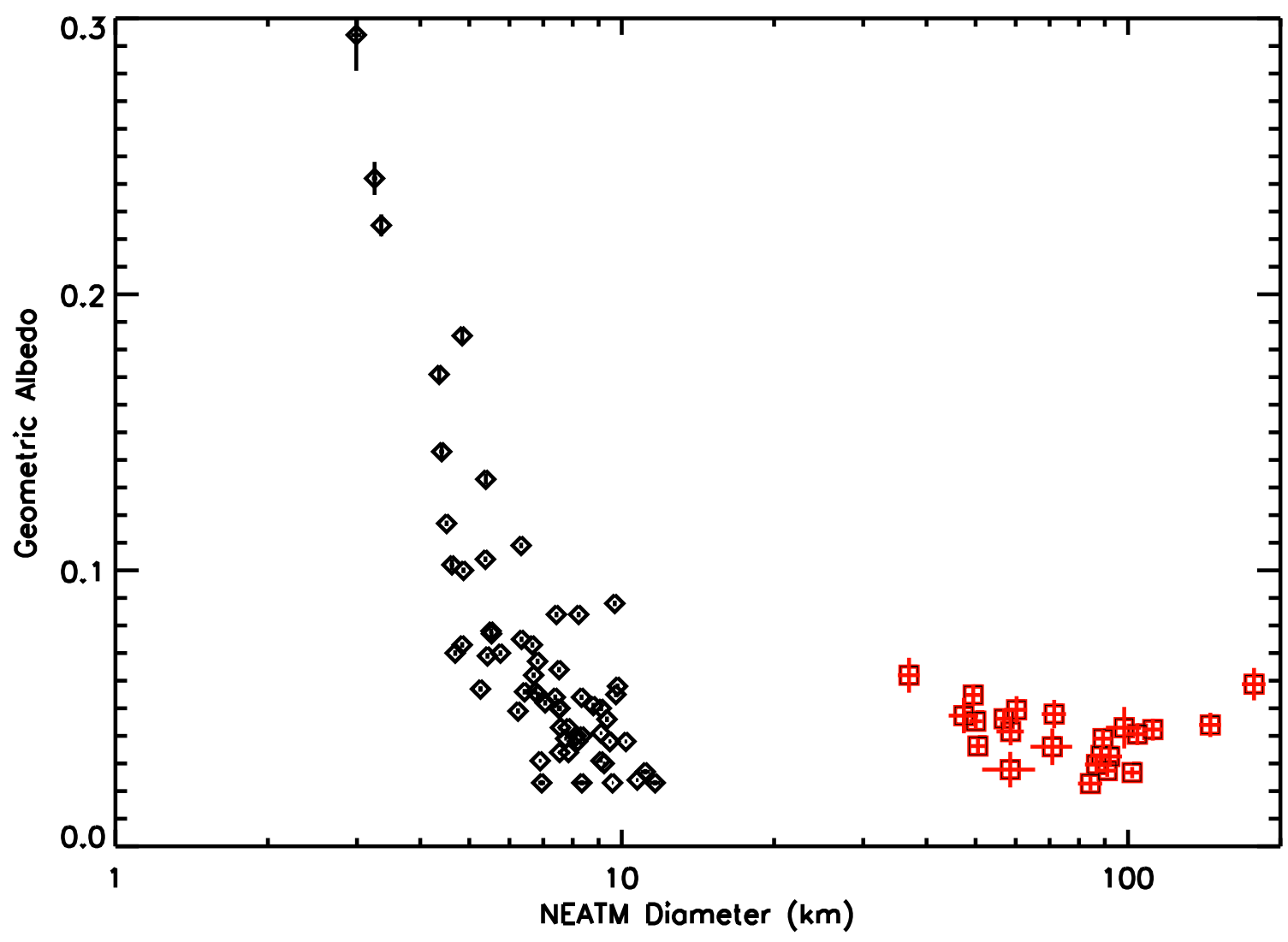

Fig. 2.- Albedo as a function of diameter for Hilda asteroids in IRAS (squares) and from Spitzer (diamonds) and corresponding error bars. 


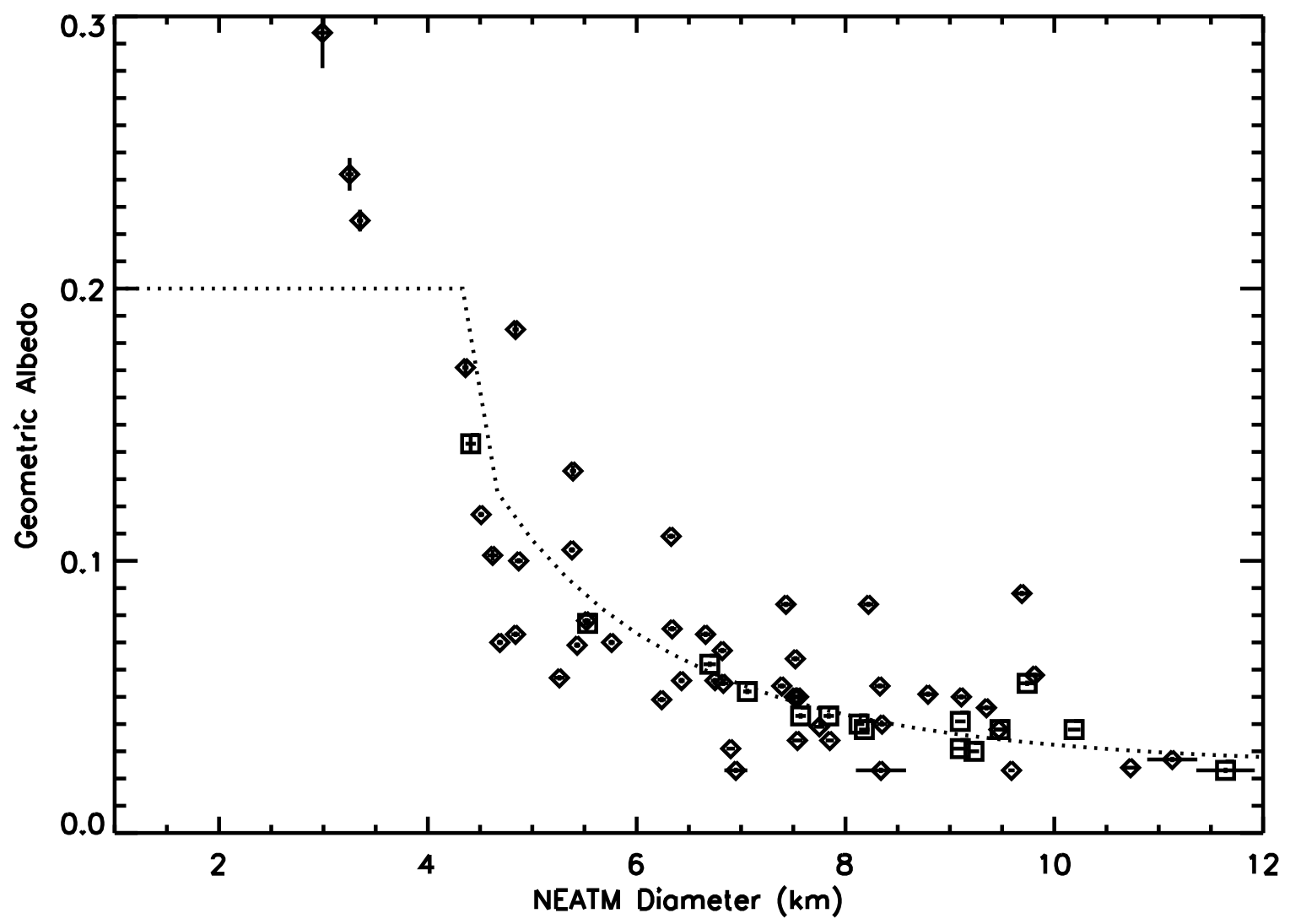

Fig. 3.- Albedo as a function diameter for Hilda asteroids in Spitzer data. Diamonds denote Hilda 'field' asteroids and squares are members of the Schubart dynamical family (see $₫ 4.4$. The dotted line represents the albedo-diameter relation as described in $\$ 4.3$. 

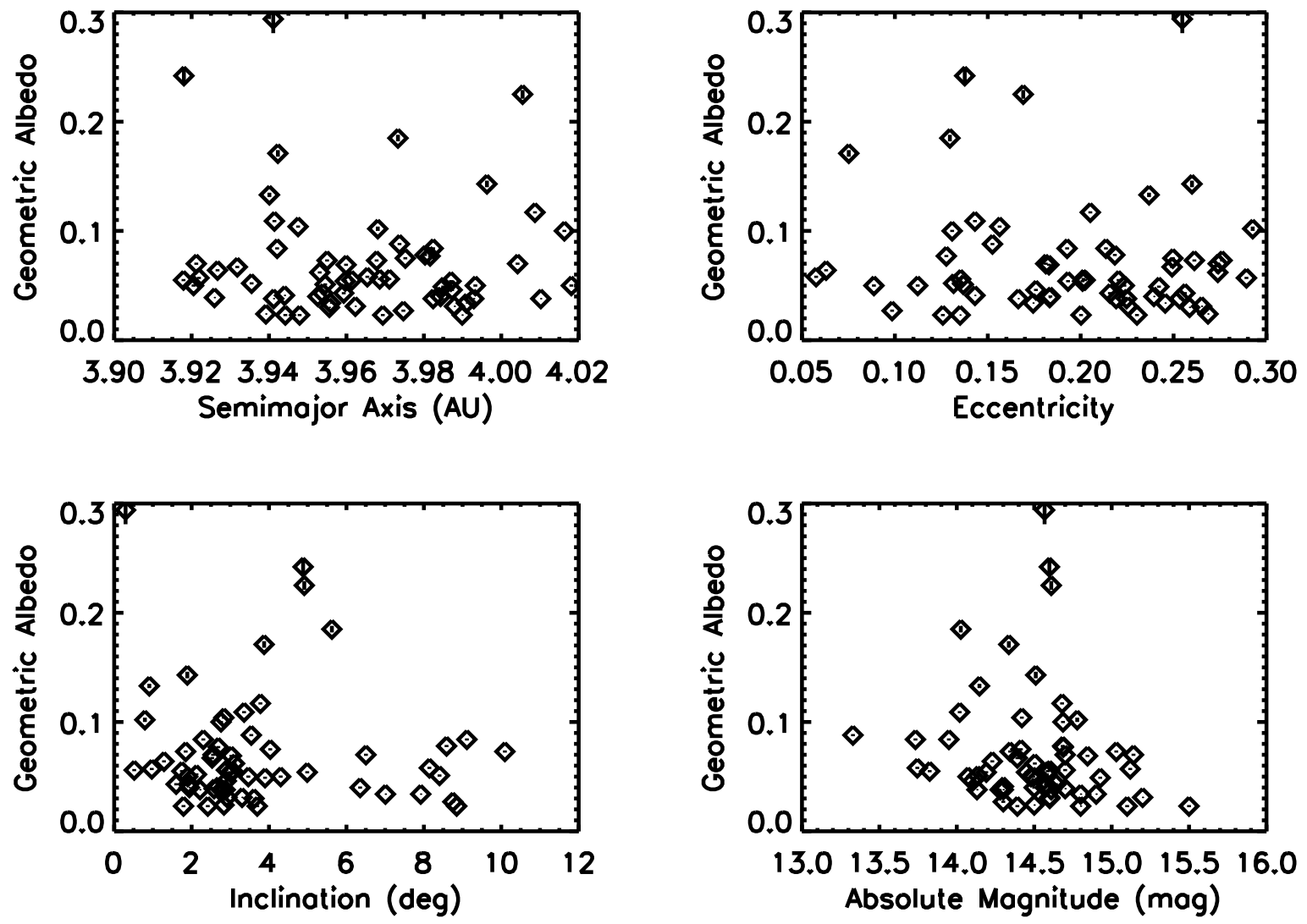

Fig. 4.- Spitzer only NEATM derived geometric albedos $p_{V}$, as function of semi-major axis, eccentricity, orbital inclination and absolute magnitude $(\mathrm{H})$ for members of the Hilda asteroid group deteced in the MIPS $24 \mu \mathrm{m}$ survey. 

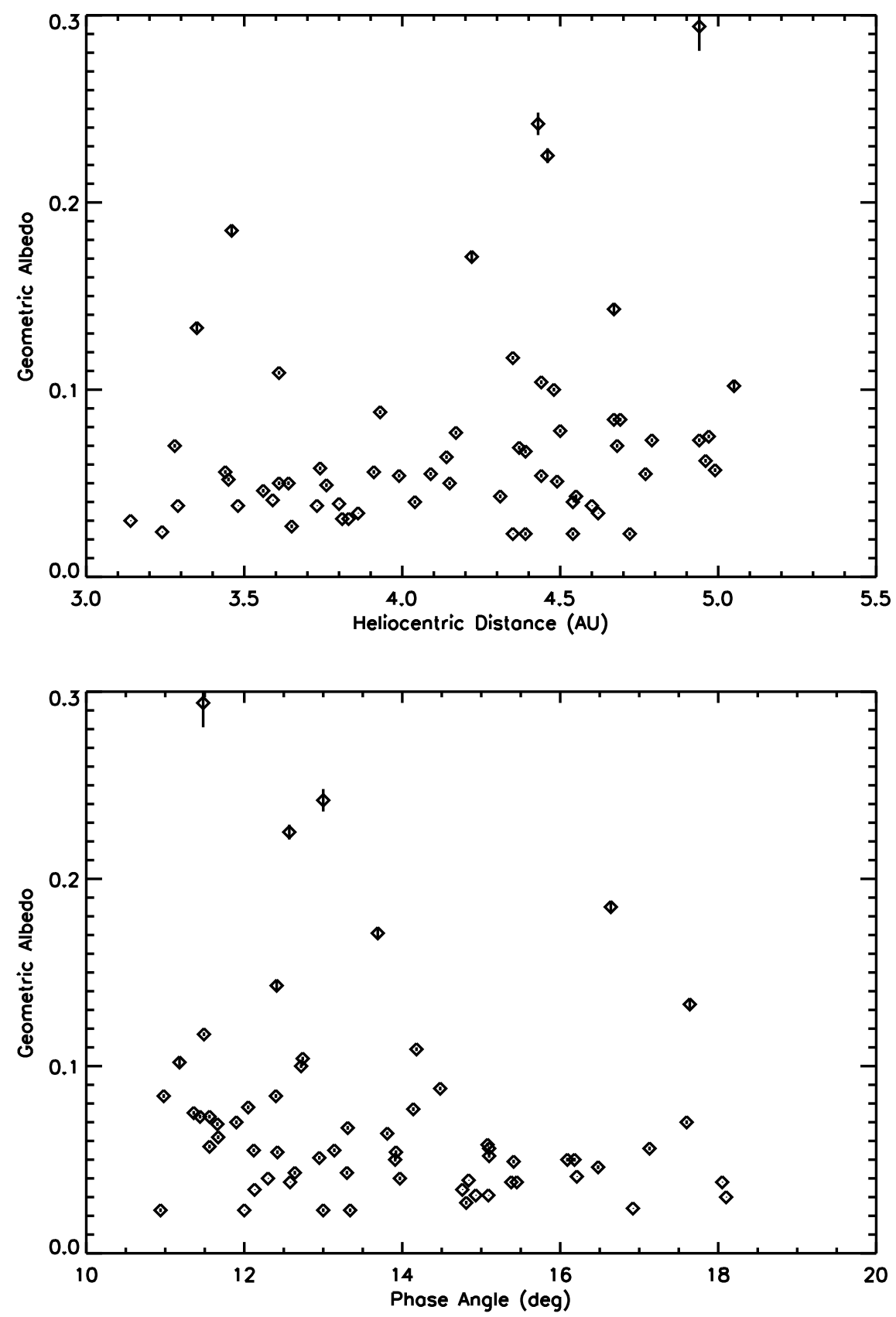

Fig. 5.- Spitzer only NEATM derived albedos as a function of heliocentric distance and phase angle for members of the Hilda asteroid group deteced in the MIPS $24 \mu \mathrm{m}$ survey. 


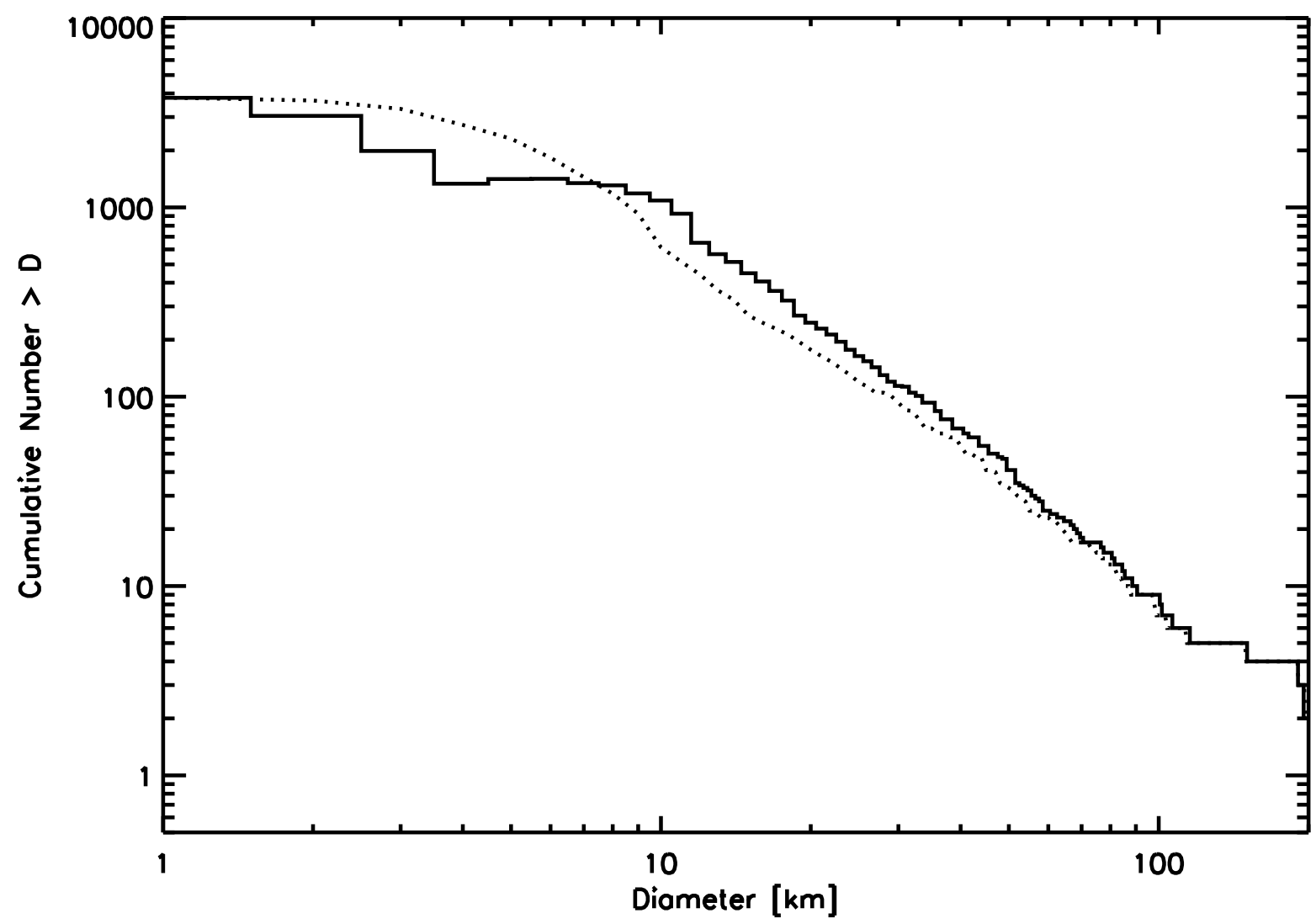

Fig. 6.- Cumulative size frequency of Hilda asteroid group utilizing the albedo function as derived from our Spitzer MIPS $24 \mu \mathrm{m}$ data (solid line) and an albedo of 0.04 (dotted line) corresponding to the average from the large Hildas detected with IRAS (Ryan \& Woodward 2010). 